\title{
Marking 15 years of the Genetic and Rare Diseases Information Center
}

\author{
Janine Lewis ${ }^{\mathrm{a}, *}$, Michelle Snyder ${ }^{\mathrm{a}}$ and Henrietta Hyatt-Knorr ${ }^{\mathrm{b}}$ \\ ${ }^{\mathrm{a}} \mathrm{ICF}$, Rockville, MD, USA \\ ${ }^{\mathrm{b}}$ National Center for Advancing Translational Sciences, Office of Rare Diseases Research, \\ National Institutes of Health, Bethesda, MD, USA
}

\begin{abstract}
.
BACKGROUND: The Genetic and Rare Diseases Information Center (GARD), a program of the National Center for Advancing Translational Sciences, was established in 2002 to assist the public in finding reliable, timely, and easy-tounderstand information about genetic and/or rare diseases in English or Spanish.

OBJECTIVE: A review of longitudinal data on GARD inquiries from 2002 to 2016 and assessment of the results of two user satisfaction surveys were conducted to understand the demographics and needs of GARD customers over time.

METHODS: Since 2002, GARD has collected anonymized data while responding to questions received via e-mail, website, telephone, fax, letter, or TTY. Between 2002 and 2016 GARD received a total of 60,106 inquiries. User satisfaction surveys were conducted in 2006 and 2014, in which users self-selected to participate.

RESULTS: The annual number of inquiries has risen steadily since 2002. Inquiries are overwhelmingly from educated female patients, family, and friends seeking disease-specific information, treatment options, referrals, and research studies. Most users report satisfaction with the experience.

CONCLUSIONS: Rare disease patients and their families face challenges in finding information about their symptoms or diagnosis, prognosis, treatment options, significance for family members, and research opportunities. Lack of available clinical expertise can leave patients, their family, and friends with little choice but to become knowledgeable on their own. GARD fills a critical need by providing the public with vetted, evidence-based information that empowers people to engage in their own health care and seek research studies of relevance.
\end{abstract}

Keywords: Health information search behavior, online information, research participation, Genetic and Rare Diseases Information Center, National Center for Advancing Translational Sciences

\section{Introduction}

Patients and families of individuals with rare diseases, which are often genetic and sometimes difficult to diagnose, can face considerable challenges in finding information about their symptoms or diagnosis, prognosis, treatment options, and significance for family members. As defined in the Orphan Drug Act, a rare disease is one that affects fewer than 200,000 people in the United States at any given time [1]. To date approximately 7,000 rare diseases have been identified as likely meeting this definition, affecting up to 30 million Americans, of which more than half are thought to be children [2].

\footnotetext{
${ }^{*}$ Corresponding author: Janine Lewis, ICF, 530 Gaither Rd, Suite 500, Rockville, MD 30850, USA. Tel.: +1 301407 6668; Fax: +1 301251 4911; E-mail: Janine.lewis@icf.com. 
Because rare diseases, by definition, are uncommon, patients and family members often are not able to obtain sufficient information from their usual sources of health information, including primary health care providers or local clinics, which might have seen few, if any, patients with their disorder. This lack of available clinical information provides patients and their family or friends with little choice but to become knowledgeable themselves. On a more positive note, research has shown that seeking health information encourages individuals to engage more actively in their health decision making [3].

The Internet has become the leading tool for the public to search for and obtain health and medical information, especially for rare disorders [4]. A 2012 national survey of more than 3,000 U.S. adults conducted by the Pew Research Center's Internet and American Life Project found that 1 in 3 Americans used Internet resources to understand a medical condition [5]. Online access to health information can also benefit individuals challenged by various access barriers to find the information they need. In a 2014 study using data from 27,210 adults from the 2009 National Health Interview Survey, Bhandari et al. found that "individuals with financial barriers to healthcare access, with difficulty in getting a timely appointment with doctors, and with conflicts in scheduling during clinic hours are more likely to search for general health information online than those without these access barriers" [6]. Thus, searching the Internet and social media can be a first step in someone's journey to find information about their own or a family member's or friend's disease, whether rare or common. Similarly, the Internet or social media can be a supplemental source of information, bolstering or complementing information provided by health care providers.

In the near future, the application of precision medicine will likely lead more people to seek information related to emergent research findings. Precision medicine will bring research findings about individual variability in genes, environment, and lifestyle into all areas of health and health care. It will disassemble common chronic diseases into multiple variations based on unique mutations [7, 8]. Clinicians and patients will increasingly use genetic and molecular information in routine health care management. However, the information can be complex, nuanced, or incomplete, requiring further exploration by patients and health care providers alike. An increase in personalized genomic information will alter the ways that the public and health care providers think about screening and prevention, which will further challenge health literacy and competency. Although research is gradually providing deeper understanding of the genetic basis of disease, we could face shortages in the number of clinical geneticists needed to respond to the public's growing interest in genetic testing and screening [9-11]. Thus, the need for reliable sources of information and links to potential referrals and research studies will be especially necessary.

Patients coping with the stress of a diagnosis of a chronic disease, rare or common, find benefit in sharing their concerns through patient groups $[12,13]$. Opportunities for these interactions can be found, initiated, and sustained through the Internet and social media. Patients can also be the most effective advocates for research on their diseases. Disease-specific patient groups are a critical source of expertise for rare diseases, and they can serve as a trusted broker between the research community and the patient population. Patients are now seeking a more active role in clinical research study design and are increasingly recognized as equal partners in the clinical trial enterprise [14-16]. This is particularly important for rare diseases for which it can be challenging for patients to find studies and for investigators to find a statistically sufficient number of patients to study. Some believe that the Orphan Drug Act of 1983 helped galvanize patient groups to become more involved in all aspects of the development and commercialization of treatments for orphan/rare conditions [17]. Online and call-in resources provide a crucial opportunity for patients and their families to find and connect with affinity groups. 


\section{The Genetic and Rare Diseases Information Center}

The Genetic and Rare Diseases Information Center (GARD) at the National Institutes of Health (NIH) was established in 2002 to assist the public_-including patients, family members, and health care providers - in finding reliable and timely information about genetic and/or rare diseases, which might otherwise not be available [18].

In 2011, GARD was relocated to the National Center for Advancing Translational Sciences (NCATS). GARD resides within the NCATS Office of Rare Diseases Research (ORDR), which facilitates and coordinates research on rare diseases, and supports a variety of programs to serve the needs of patients who have any one of the many rare diseases known today. GARD receives funding through NCATS and the National Human Genome Research Institute (NHGRI) to help patients, their families, health care professionals, researchers, and the public locate timely and reliable information.

Since its inception 15 years ago, GARD has worked to gather, archive, and make available information on rare diseases. People coming to GARD can interact with information specialists by phone, e-mail, web inquiry, letter, fax, or TTY. Information specialists either direct inquirers to existing materials available on the GARD website or assist in locating additional current, accurate, and authoritative information from other resources. Information specialists provide information drawn from public domain sources, such as reliable websites, brochures, medical literature, lay articles, or patient organizations.

On the GARD website, each disease has its own webpage, where information specialists post the answers to questions GARD has received. Webpages also contain links to resources with information about genetic testing and genetic services, NIH-sponsored scientific conferences, patient groups, research studies and clinical trials, and Food and Drug Administration (FDA)-approved medications for rare diseases. Through this public resource, GARD users can search for information on more than 6,700 genetic and/or rare diseases and pose questions directly to an information specialist.

GARD has made many changes in its information delivery over the past 15 years. GARD created an online database of disease-specific information in 2008 to enable visitors to conduct their own searches for information about genetic and/or rare diseases. Since 2008, changes have been made to the website based on usability testing and feedback provided voluntarily by visitors to the site. Users can find information about a disease through the search filters, alphabetical disease list, or disease categories (e.g., eye diseases, skin disorders). If a visitor is unable to find the name of the condition using the search function or needs additional information, he or she can contact an information specialist at GARD via multiple channels including phone or e-mail. In addition to providing disease-specific information, the GARD website contains cross-cutting information such as how to find a disease specialist, tips for the undiagnosed, and financial aid resources, topics that the public frequently ask questions about.

Since GARD was established, the information center has responded to a changing environment in which the Internet has become the leading platform for delivering health information. To continuously improve its services, GARD tracks its inquiries and passively collects some anonymized data, for example: role such as patient, family member, or health care provider; frequency of use of GARD; referral; and country of origin or U.S. state. Any identifying information needed to respond to the inquirer is deleted 60 days after the initial request is received. The anonymized information that is collected facilitates a better understanding of the demographics and needs of GARD customers. In 2006 and 2014, user satisfaction surveys were another means of evaluating responsiveness to the public. Below are summaries of these longitudinal analyses of GARD inquiries and user satisfaction. 


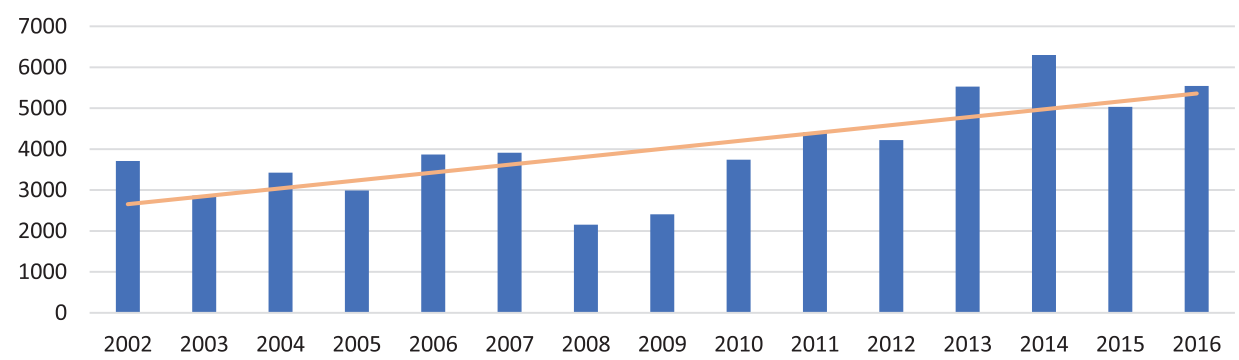

Fig. 1. GARD inquiries received annually, 2002-2016. The number of inquiries GARD received each year via all channels including phone, email, online form, letter, fax, and TTY.

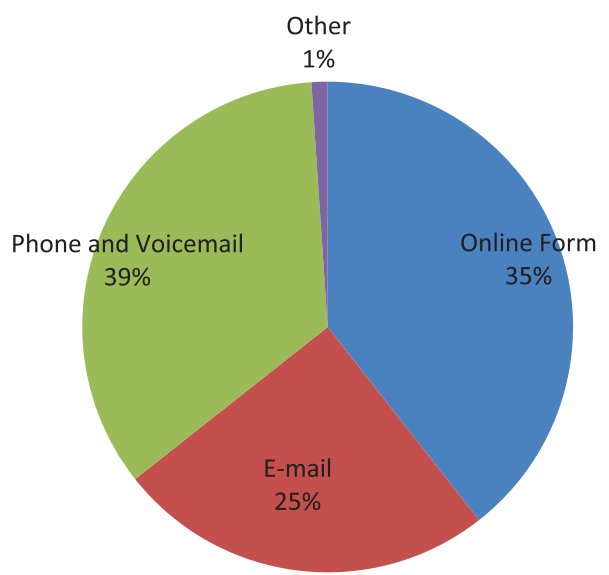

Fig. 2. Cumulative GARD inquiries by mechanism, 2002-2016. The method inquirers used to contact GARD with their questions.

\section{Analysis of data, 2002-2016: What we know about GARD inquiries}

\subsection{Volume of inquiries}

Since 2002, GARD has collected anonymized data about inquiries while responding to questions received via e-mail, the GARD website, telephone, or other means, such as fax, letter, or TTY. From 2002 until the end of 2016, GARD received a total of 60,106 inquiries, with the number of inquiries increasing steadily each year, growing from 3,711 in 2002 to 5,542 in 2016 (Fig. 1).

\subsection{Mechanisms of inquiry}

GARD receives inquiries over the phone and in writing. Inquiries by phone accounted for $40 \%$ of cumulative interactions over the 15 years, with written contact accounting for a cumulative $60 \%$ (Fig. 2). In 2016, many users continued to contact GARD by phone: $49 \%$ of inquiries were via phone or voicemail. Phone inquiries come from people with and without Internet access and provide patients and family members the opportunity to speak with someone about their concerns and ask questions.

Since May 2013, GARD has used a consistent web analytics platform to track the number of users who access the website. There has been an increase in recent years. The number of unique users has increased from 177,366 in May 2013 to 389,740 in December 2016 (Fig. 3). GARD redesigned its website in August 2016 to make it more accessible, which could explain the uptick in web traffic occurring in late 2016. 


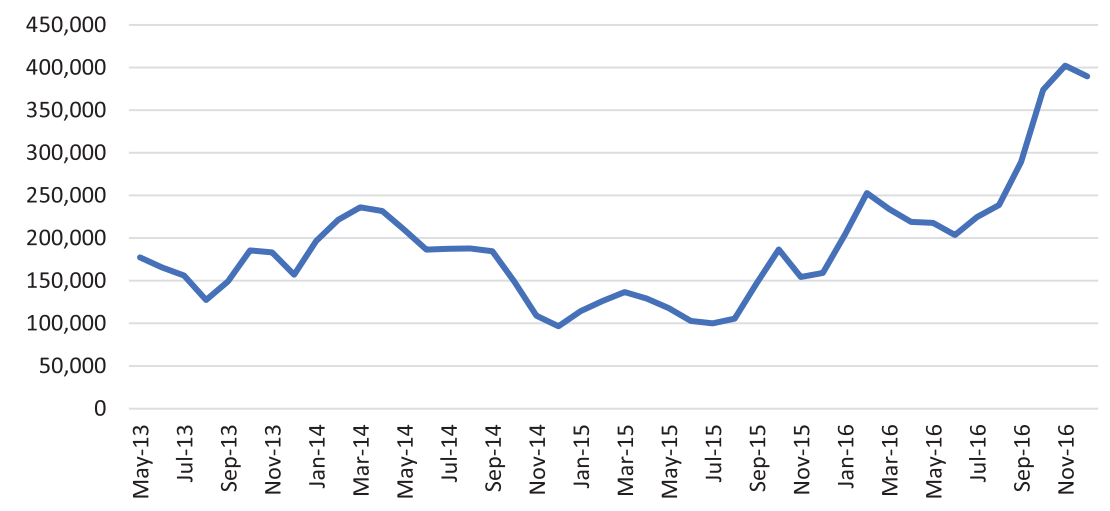

Fig. 3. Number of GARD website users. The number of unique visits to the GARD Website by month since May 2013.

\subsection{Demographic data on inquirers}

GARD passively captures anonymized information about those who contact the service. Most basic demographic information (e.g., age, state of residence, race/ethnicity) is passively collected, so it tends to be available for only a fraction of all inquiries. Information about inquirers' gender, primary language, and country of origin are captured for most inquiries.

Inquiries were nearly always conveyed in English, with only 3\% of inquiries in Spanish. Seventy-six percent of inquiries are from within the United States. Consistent with other data on health-seeking behavior by the public, females make inquiries at a greater rate than males. Since 2002, approximately $58 \%$ of inquiries have come from females and $19 \%$ from males (23\% of inquiries did not indicate gender). In 2016, $17 \%$ of inquiries were from males. These data are highly consistent with studies showing that women tend to be the primary seekers of health information for their families, as well as for themselves [19]. A 2011 study of Internet health searching by the Pew Research Center found that women significantly outperform men in their quest for information about specific diseases or medical problems, treatments, procedures, and searches for doctors or other health professionals or medical facilities [20].

Inquiries overwhelmingly come from patients (34\%) and from family and friends (37\%) (see Fig. 4). Health care professional inquiries account for $8 \%$, and $5 \%$ of questions come from educators and students. The remainder are either not specified (11\%), other professionals (e.g., social workers, lawyers; $3 \%$ ), and researchers and patient advocacy groups (each at 1\%). These proportions have remained relatively steady over time.

Data from the 2014 user satisfaction survey generally parallel the findings from the longitudinal data (see more below). In 2014, the principal characteristics of survey respondents were:

- Patient $(41 \%)$ or family member/friend of a patient $(25 \%)$

- Female $(76 \%)$

- Age 51-60

- Bachelor's degree (25\%); Master's degree (17\%); Doctoral/Professional degree (13.2\%)

- White $(86 \%)$

- Prefers to speak and read in English (90\%)

- Resides in a suburban area $(40 \%)$

- If resided in the United States (80\%), resided in the Census-defined South (35\%)

- Of the $20 \%$ residing outside of the United States, 35\% reside in Europe 


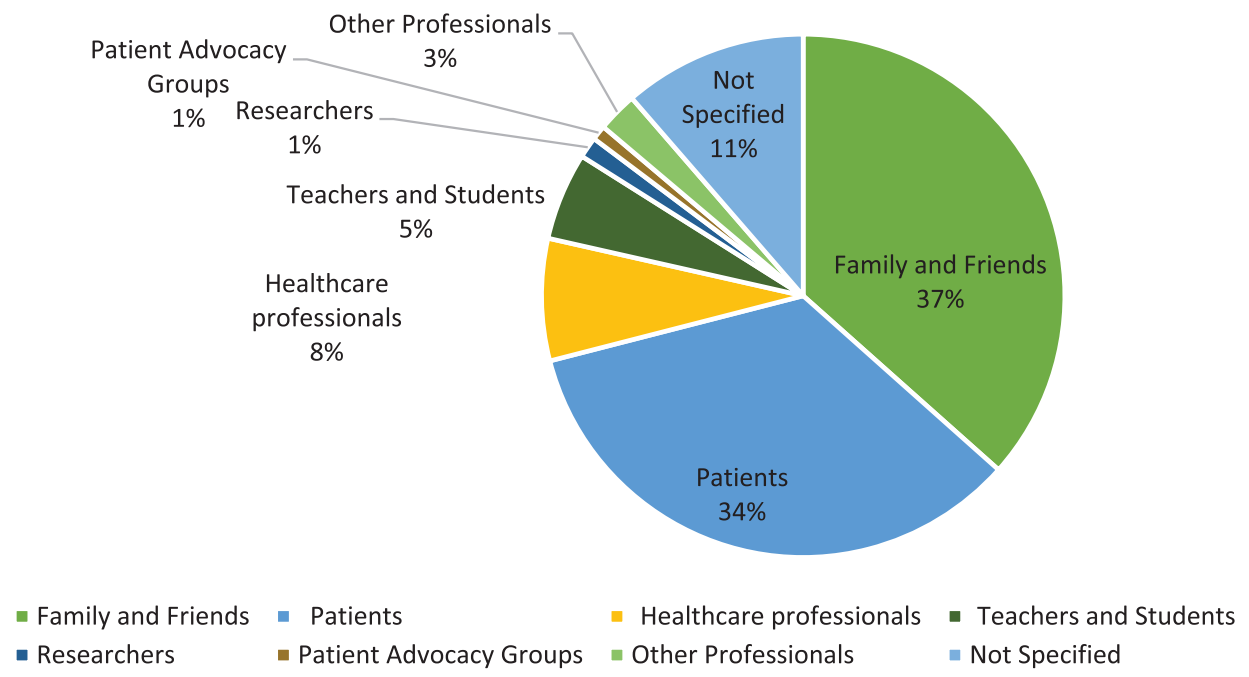

Fig. 4. Cumulative GARD inquiries by user category, 2002-2016. The user categories of the people who contacted GARD over 15 years. The majority $(71 \%)$ are patients, their family members and friends. The "not-specified" category represents people who did not identify what their role is with regard to the question(s) they are asking.

Table 1

Top 5 reasons for GARD inquiries, 2002-2016 $(N=60,106)$

\begin{tabular}{lc}
\hline Reason & Number (\% of Total) \\
\hline Treatment questions & $11,891(20 \%)$ \\
Seeking a physician referral & $7,551(13 \%)$ \\
Seeking a research study & $5,137(9 \%)$ \\
Undiagnosed (symptoms provided but no diagnosis) & $4,583(8 \%)$ \\
Clinical/genetic testing & $4,068(6 \%)$ \\
\hline
\end{tabular}

Four out of five inquirers $(81 \%)$ want information about a specific disease. These topics represent the top 5 aspects of specific diseases about which people ask.

Some of the questions asked in the 2014 user satisfaction survey were also asked in the 2006 survey. The demographic characteristics of the convenience sample who completed the 2006 survey were similar to those who completed the 2014 survey except for the following. The top bracket for age of inquirers rose from 31-40 in 2006 to 51-60 in 2014, possibly suggesting greater access to and use of the Internet by older individuals.

\subsection{Reasons for inquiries}

Inquiries are coded with one or more reasons based on the topics requested. Eighty-one percent of inquiries pertained to a specific disease. Inquiries that were not disease-specific included requests for general information on rare disease statistics or financial resources, for example. Table 1 displays the top 5 reasons for disease-specific inquiries from 2002 to 2016. Twenty percent of inquiries were about treatment options or referral to a specialist in the condition (13\%).

Inquiries seeking information on treatment and referrals have steadily increased over time (Fig. 5). GARD information specialists direct individuals seeking referrals to patient groups, published resources, and the medical literature to find clinical specialists and treatment centers. GARD provides a guide called "How to Find a Disease Specialist" on its website or as a brochure and clearly 


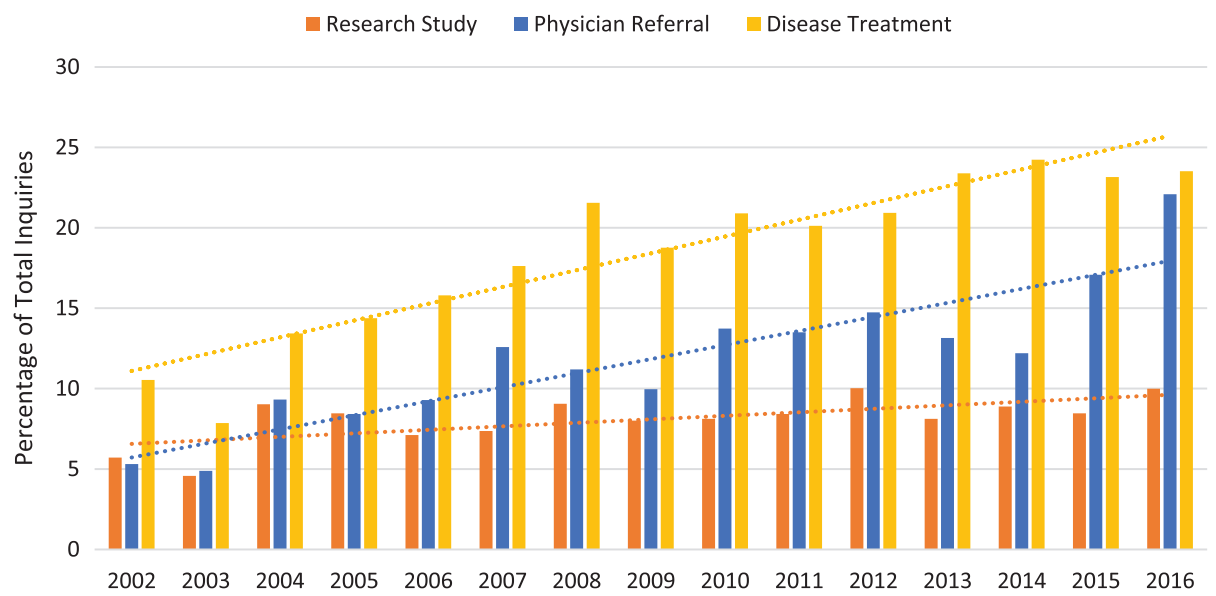

Fig. 5. Top three reasons for GARD inquiry, 2002-2016. These are the topics for which people have asked about the most about over 15 years. There has been a significant increase each year in questions from people looking for treatment information and for help finding an expert in their disease. Questions about learning more about or participating in research have remained relatively steady.

states that it provides these resources for informational purposes only and not as an endorsement of services. GARD information specialists do not provide genetic counseling or medical advice; rather, they steer the inquirer to appropriate sources of information about such services when appropriate.

Interest in research studies has been a steady focus of GARD inquiries. It is the third leading reason for inquiry for the years 2002 to 2016. As with questions about referrals to specialists, GARD staff direct individuals to resources to learn about research studies and how to participate in research. Despite the growing interest in treatment and referrals, inquiries about clinical, genetic, and prenatal testing are the fifth leading topic of inquiry between 2002 and 2016, accounting for 1 out of 15 of all incoming inquiries and remaining relatively stable at that level each year from 2002 to 2016.

\subsection{Referrals to GARD}

Google was named as the top referrer for web traffic in the past 2 years (70\%). This percentage is consistent with the results of previous surveys, which found that $64 \%$ of respondents learned about GARD by using an Internet search engine. For very rare conditions, the GARD Information Center link is often listed as one of the top search results because of limited information available from other sources.

\subsection{Diseases and conditions cited in GARD inquiries}

Inquiries cover a wide range of diseases and conditions. GARD information specialists have researched 4,265 different conditions from 2002 to 2016. The number of inquiries received about each disease is usually very small, with a median of three inquiries received per disease. Table 2 shows the top 20 diseases and conditions requested in GARD inquiries. To demonstrate the wide-ranging nature of these disease inquiries, the top specified disease, trimethylaminuria, represents only $1 \%$ of total inquiries received.

The diseases and topics that rise to the top of the list do not necessarily reflect disease prevalence. It is more likely that the relative popularity of various rare diseases among inquiries reflects other factors and trends, such as the amount of information available elsewhere on the Internet, or current public or scientific media focus on a given disease. 
Table 2

Leading diseases and topics of inquiries, 2002-2016 $(N=60,106)$

\begin{tabular}{lc}
\hline Disease or Condition & Number \\
\hline Undiagnosed condition & 4,436 \\
Trimethylaminuria & 659 \\
Morgellons disease & 310 \\
Ehlers-Danlos syndrome & 288 \\
Adiposis dolorosa & 239 \\
Familial Mediterranean fever & 230 \\
Hidradenitis suppurativa & 228 \\
Cystic fibrosis & 223 \\
Factor V Leiden thrombophilia & 212 \\
Klinefelter syndrome & 185 \\
Tarlov cysts & 176 \\
Sickle cell anemia & 171 \\
Huntington disease & 162 \\
\hline
\end{tabular}

Of the 60,106 inquiries GARD has received over 15 years, these are the diseases about which GARD has received the most questions. GARD has answered questions about more than 4,200 diseases.

\section{GARD user satisfaction}

GARD has conducted two consumer satisfaction surveys to systematically collect data to support changes to the information service, and to ensure it continues to operate in a cost-effective manner and to provide an effective, high-quality service to a broad audience.

\subsection{Survey results}

The first Office of Management and Budget (OMB)-approved survey in English and Spanish was conducted in 2006, prior to the establishment of the website. The overall response rate was $35.4 \%$ $(N=230)$ from both e-mail and telephone. Results found that:

- Information center customers were white, non-Hispanic, English-speaking females between the ages of 31 and 40 who have a post-graduate education.

- Most respondents identified themselves as a family member or friend of a patient and were seeking information about a specific disease or condition, of which they had done research prior to contacting the information center.

- Most respondents reported getting their health information over the Internet, and first learned about the information center by conducting a search using an Internet search engine, such as Google or Yahoo.

- Respondents thought the responses they received from the information center were effective.

- Overall, respondents were very satisfied with the responses they received and with the information specialists (for those who had direct contact with the information specialists). Most respondents would definitely contact the information center again and would definitely tell others to contact the information center.

- Greater than $50 \%$ of respondents have shared or plan to share the information they received from the information center with family members of friends. 


\subsection{Survey results}

In 2014, a web-based survey with OMB approval was developed in English and Spanish. This survey contained 29 questions and was designed to identify: (1) who is being served by GARD; (2) how individuals learn about GARD; (3) why individuals seek information from GARD; (4) the type of information being sought; (5) where else customers might have sought information and assess customers' satisfaction. Twenty-seven questions were closed-response (i.e., pre-coded) and two were open-ended.

Data collection commenced on March 10, 2014, and ended April 25, 2014. Individuals who contacted GARD by visiting its webpages, submitting the online contact form, sending an email message or letter, or calling the Center self-selected to complete the survey in English or Spanish. Individuals who contacted GARD by phone were invited to complete the survey by phone or online. During the data collection period, 930 surveys were completed of which $98 \%$ were completed in English and $2 \%$ in Spanish.

Survey respondents who received information by speaking with a GARD information specialist on the telephone expressed being highly satisfied with the information they received (as indicated by approximately $33 \%$ to $58 \%$ of respondents answering completely 8 of the 10 questions related to satisfaction). Survey respondents were most likely to share the information they received from GARD with others, such as a health care provider, family member, or co-worker, or use the information to educate themselves or for educational reports or presentations.

\section{Discussion}

Since 2002, GARD has witnessed steady growth in the number of inquiries it receives each year. Even with the growth in Internet access and the expansion of GARD's web presence in 2008, members of the public continue to telephone the center, even when they have access to the Internet. Reasons for this may include a need to interact with and share concerns and voice questions with a knowledgeable person. Callers also contact GARD with questions about information they have viewed on the GARD website, which might not answer all their questions or could raise additional questions. Even though web access has increased access to GARD information, and web traffic has increased since GARD started tracking it, it is important to maintain the capacity for phone and voicemail contact. Results from the user satisfaction surveys show that users who received responses through the telephone were more likely to be highly satisfied with their experience than those seeking and receiving their responses electronically.

Both the longitudinal data and the user satisfaction surveys confirm that patients $(34 \%$ in the cumulative data and $41 \%$ in the user satisfaction survey) and their friends and their families (37\% in the cumulative data and $24.7 \%$ in the user satisfaction survey) comprise the majority of inquirers. And consistent with data from other health-seeking analyses, females and those identifying as white account for a substantial majority of inquiries. Users are more likely to have completed college or graduate education. Of note, professions such as physicians, nurses, research scientists, educators, students, and members of the media are less likely to make inquiries (18\% combined from longitudinal data and $13 \%$ combined from the user satisfaction survey). This highlights the importance of the GARD service in educating the general public about genetic and rare disease. Most users arrive at the GARD website through a commonly used search engine such as Google or Yahoo, which again emphasizes the self-help aspects of these inquiries. Furthermore, users report in the surveys that they often share the information they receive with others, such as a health care provider or family member. 
Nearly every user has a question about a specific disease. And while inquiries covered 4,265 different conditions since 2002, there remain many diseases for which information is needed but exceedingly difficult to find. Such users rely on the expertise of the highly trained GARD staff to find available resources, as scarce as they might be. Many inquiries come from individuals with an undiagnosed condition, suggesting they are seeking a diagnosis and exploring possibilities on the GARD webpages. This underscores again the difficulty people with rare diseases have in obtaining a diagnosis and the long diagnostic odyssey patients often encounter.

The reason for the increase in the proportion of users seeking a referral is not clear and deserves further exploration. This trend underscores the substantial need of patients with rare diseases for clinical services not available in their immediate area. When people are more engaged to learn more about their health condition online, it can promote the desire to connect directly with an experienced health care professional. The increased interest in treatment information tracks with the growing interest in seeking a referral.

Of note, the proportion of GARD users seeking research studies has also increased steadily from 2002 and 2016 and was the fourth top reason for inquiry identified by respondents to the 2014 user satisfaction survey. Almost 1 in 10 inquirers in that survey were interested in participating in research. Patients with rare diseases often benefit from research studies that also provide the standard of care as part of the study. GARD users might find the same geographic and financial barriers to accessing care as they do to research participation if they do not reside near an academic medical center with the needed clinical and scientific expertise.

Results from the user satisfaction surveys show that users who received responses through the telephone were more likely to be highly satisfied with their experience than those seeking and receiving their responses electronically. In addition, GARD frequently receives messages of appreciation from users in the form of phone calls and e-mail messages.

The findings presented here are consistent with those of a 2014 study of individuals seeking information about rare and/or genetic diseases using a random sample of GARD inquiries [21]. Conclusions suggested "that lay people contacting a genetic and rare disease information center most often seek information about disease prognosis, finding a specialist, and obtaining a diagnosis for symptoms." This review also found a growing interest in participating in clinical research and in the intention of visitors to supplement or better understand information received from a health care provider. Patients were the most common category of inquirers, according to the study authors, possibly revealing "the extent to which patients with genetic and rare diseases act as their own health care advocates."

Finally, there are limits to these data. The user satisfaction surveys rely on people who self-select, who might be more vested or involved in their health care decision making. Furthermore the longitudinal data are not complete and do not reflect the vast number of users for which no data are collected. Despite these limitations, the consistency of data across several sources suggests GARD has a general sense of the attributes of its users and their reasons for inquiry.

\section{Conclusions}

Since its inception 15 years ago, GARD has served thousands of patients, their families, and health professionals. This service has unfolded against a backdrop of exciting developments in the fields of genomics and genetic and rare diseases research, bringing hope to many of a better understanding of rare disease etiology and, ultimately, of improved treatments. As these developments continue to evolve, people with rare diseases will continue to need an easily accessible and authoritative information resource that can provide rapid, accurate, and empathetic answers to complex questions. 
GARD believes in the importance of building partnerships with the health care community. Information specialists always strongly recommend that patients seek the advice of a health care provider about any questions regarding their care. Health care providers can also use GARD's services as a timesaving tool for accurate, up-to-date information on genetic and rare diseases.

Access to accurate and targeted information empowers people to engage more actively in their own health care decision making. It can also lead them to promising research studies. Capturing the patient's voice is a welcome trend in medicine to integrate patient-defined values and needs into evidencedbased research and clinical practice. For the more than 25 million people living with a rare disease, this trend is especially critical. With scarce dollars for rare disease research and small cohorts, it is more important than ever to design study protocols that have examined the influence of disease context and patient's benefit-risk preferences.

Informing the patient and amplifying his or her voice will move rare disease research forward, translating observations in the laboratory, clinic, and community into interventions that improve health. As GARD moves into the future, increased efforts are needed to assess and meet unmet needs of the rare disease community. GARD is aware that there are "difficult to reach" groups that are under resourced, geographically isolated, or lacking the skills needed to seek and find the answers to their health questions. Current GARD users are not as diverse as desired in terms of racial/ethnic diversity and educational attainment. Relatively low use by health care professionals suggests different mechanisms are needed to target that population. GARD is exploring how to address these concerns while continuing to respond to an ever-increasing public need for information on rare and genetic diseases.

\section{References}

[1] Genetic and Rare Diseases Information Center. Frequently asked questions; n.d. https://rarediseases.info.nih.gov/aboutgard/pages/31/frequently-asked-questions.

[2] The Global Genes Project. RARE Diseases: Facts and Statistics; 2015. http://globalgenes.org/rare-diseases-factsstatistics/.

[3] R. Bratucu, I. Gheorghe, R. Purcarea, C. Gheorghe, O. Popa Velea and V. Purcarea, Cause and effect: The linkage between the health information seeking behavior and the online environment-a review, J Med Life 7(3) (2014), $310-316$.

[4] J.G. Hamilton, S.P. Hutson, A.E. Frohnmayer, P.K. Han, J.A. Peters, A.G. Carr and B.P. Alter, Genetic informationseeking behaviors and knowledge among family members and patients with Inherited Bone Marrow Failure Syndromes, J Genet Couns 24(5) (2015), 760-770. doi:10.1007/s10897-014-9807-3

[5] S. Fox and M. Duggan Health Online 2013. Pew Internet \& American Life Project; 2013. http://pewinternet.org/ Reports/2013/Health-online/Summary-of-Findings.aspx

[6] N. Bhandari, Y. Shi and K. Jung, Seeking health information online: Does limited healthcare access matter? J Am Med Inform Assoc 21(6) (2014), 1113-1117. doi:10.1136/amiajnl-2013-002350

[7] The White House. Remarks by the President in the State of Union Address; Sept 25, 2015. https://www. whitehouse.gov/the-press-office/2015/01/20/remarks-president-state-union-address-january-20-2015

[8] F.S. Collins and H. Varmus, A new initiative on precision medicine, N Engl J Med 372(9) (2015), 793-795. doi:10.1056/NEJMp1500523

[9] A.E. Guttmacher, M.E. Porteous and J.D. McInerney, Educating health care professionals about genetics and genomics, Nat Rev Genet 8 (2007), 151-157.

[10] A.E. Guttmacher, A.L. McGuire, B. Ponder and K. Stefánsson, Personalized genomic information: Preparing for the future of genetic medicine, Nat Rev Genet 11 (2010), 161-165.

[11] D.S. Dodson, A.J. Goldenberg, M.M. Davis, D.C. Singer and B.A. Tarini, Parent and public interest in whole-genome sequencing, Public Health Genomics 18 (2015), 151-159.

[12] R. Overberg, W. Otten, A. de Man, P. Toussaint, J. Westenbrink and B. Zwetsloot-Schonk, How breast cancer patients want to search for and retrieve information from stories of other patients on the Internet: An online randomized controlled experiment, J Med Internet Res 12 (2010), 1. doi:http://dx.doi.org/10.2196/jmir.1215

[13] R.L. Lee and L.M. Kvasny, Understanding the role of social media in online health: A global perspective on online social support, First Monday 19(1) 2014, [in press]. doi: http://dx.doi.org/10.5210/fm.v19i1.4048 
[14] P. Wicks, T. Vaughan and J. Heywood, Subjects no more: What happens when trial participants realize they hold the power? BMJ Br Med J 368 (2014), 2-3. http://www.bmj.com/content/348/bmj.g368

[15] M. Anderson and K.K. McCleary, On the path to a science of patient input, Sci Transl Med 8(33) (2016), $336 \mathrm{ps} 11$.

[16] Clinical Trials Transformation Initiative. PG Engagement Across the Research \& Development Continuum; 2015. http://www.ctti-clinicaltrials.org/what-we-do/investigationalplan/patient-groups

[17] R. Robinson, Patients and Patient Organizations Power Rare Disease Therapies, PharmaVOICE; Feb 2016. http://www.pharmavoice.com/article/2016-02-rare-disease-therapies/

[18] L. Wang, J. Wang, M. Wang, Y. Li, Y. Liang and D. Xu, Using Internet search engines to obtain medical information: A comparative study, J Med Internet Res 14(3) (2012), e74. doi:10.2196/jmir.1943

[19] D. Warner and J.D. Procaccino, Toward wellness: Women seeking health information, J Am Soc Inf Sci Technol 55(8) (2004), 709-730.

[20] S. Fox, Health Topics, Pew Research Center; Feb 1 2011. http://www.pewinternet.org/2011/02/01/health-topics-2/

[21] T. Morgan, J. Schmidt, C. Haakonsen, J. Lewis, M. Della Rocca, S. Morrison, B. Biesecker and K.A. Kaphingst, Using the Internet to seek information about genetic and rare diseases: A case study comparing data from 2006 and 2011 , JMIR Res Protoc 3(1) (2014), e10. doi:10.2196/resprot.2916 